\title{
Persistent Localization and Life-long Mapping in Changing Environments using the Frequency Map Enhancement
}

Tomáš Krajník

Jaime Pulido Fentanes
Marc Hanheide

Tom Duckett

\begin{abstract}
We present a lifelong mapping and localisation system for long-term autonomous operation of mobile robots in changing environments. The core of the system is a spatiotemporal occupancy grid that explicitly represents the persistence and periodicity of the individual cells and can predict the probability of their occupancy in the future. During navigation, our robot builds temporally local maps and integrates then into the global spatio-temporal grid. Through re-observation of the same locations, the spatio-temporal grid learns the longterm environment dynamics and gains the ability to predict the future environment states. This predictive ability allows to generate time-specific $2 d$ maps used by the robot's localisation and planning modules. By analysing data from a long-term deployment of the robot in a human-populated environment, we show that the proposed representation improves localisation accuracy and the efficiency of path planning. We also show how to integrate the method into the ROS navigation stack for use by other roboticists.
\end{abstract}

Index Terms-mobile robotics, long-term autonomy

\section{INTRODUCTION}

One of the many challenges that robots have yet to achieve is long-term autonomous operation in changing environments. Many tasks performed by mobile robots take place in environments where humans perform their usual activities, which causes the environments to change constantly. For example, doors are open and shut, chairs are pushed in and out of tables and furniture is occasionally rearranged. In long-term scenarios, having an environment model that assumes a static world will inevitably lead to navigation failures as the robot's knowledge base becomes obsolete.

In this paper, we propose an architecture for life-long mapping and persistent localization that is easily integrated within the ROS framework [1]. Through extension of the ROS navigation stack by an additional ROS gmapping module, we obtain a system that can create an up-to-date, independent environment map on-the-fly. To enforce the compatibility of the new map with the previous environment models, we propose to use the position estimates of the ROS $A M C L$ module as virtual odometry for the gmapping node.

The newly created, independent maps are integrated into a spatio-temporal occupancy grid where each cell contains a frequency-spectrum of its past states [2] and allows the prediction of the cell's future states. Previous work has shown that the predictive capabilities of the spectral models improve visual-based mobile robot localisation [3], navigation [4] and planning [5]. However, the previous works were aimed at proof-of-concept verification of the Frequency Map

All authors are with the University of Lincoln, UK. This work was funded by the EU ICT project 600623 'STRANDS'

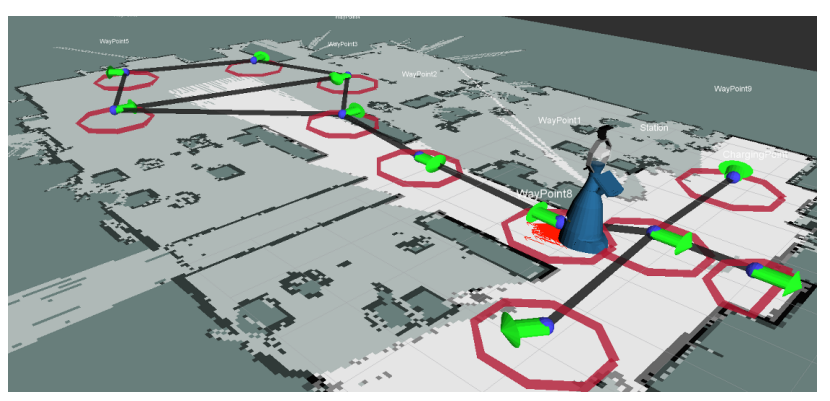

Fig. 1: Predicted and created 2D grids with patrol locations.

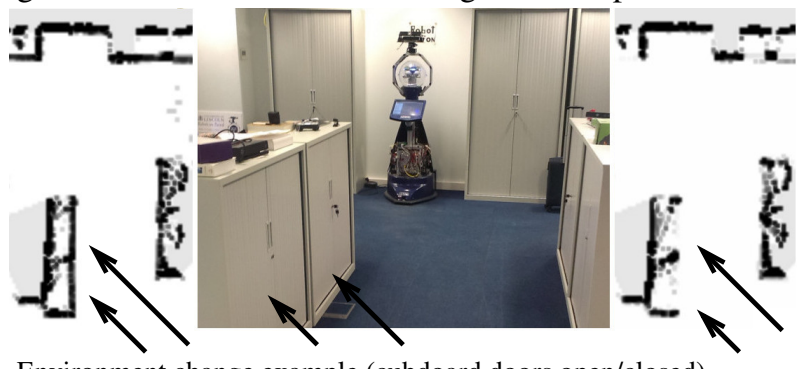

Environment change example (cubdoard doors open/closed)

Fig. 2: Example of regular changes and corresponding map predictions for early morning (left) and late afternoon (right).

Enhancement (FreMEn) methods using custom modules that were not fully integrated in the ROS navigation framework. In this work, we show that once the ROS navigation stack is extended so that it allows the creation of new maps, integration of the FreMEn is straightforward, as it simply replaces the map server ROS module.

To validate the proposed method, we set up our robot to routinely patrol a human populated office environment, which was subject to frequent changes due to the people's activity. During each patrol run, which started and ended at the robot's charging station, a new map was created and as soon as the patrol was completed, this new map was integrated into the FreMEn spatio-temporal model. At the start of each patrol, the spatio-temporal model predicted a time-specific map that was used by the robot's planning and localization methods. As the model accumulated enough data to infer the long-term environment dynamics, the predicted maps started to differ according to the time of the patrols. For example, the spatio-temporal model predicted that on weekday afternoons, doors of certain cupboards are more likely to be open than during nights, see Figure 2 .

To evaluate how the predictive capability of our method affects the efficiency of the robot operation, we provide statistics on the accuracy of the robot localization, navigation performance, planning failures and map quality. 
This paper extends the previous work on Frequency Map Enhancement [2], which allows to introduce dynamics into most robotic environment models. In particular, we show that the method can be extended to model not only cyclicperiodic changes, but also their persistence. We propose an architectural modification of the ROS navigation stack that allows for straightforward update of the environment models, which enables ROS integration of the proposed method in an elegant and straightforward way. Last, but not least, we demonstrate that the introduction of the method improves the efficiency of the robot operation in long-term scenarios.

\section{RELATED WORK}

Long-term robot navigation is highly dependant on the precision of the world model, since the robot requires such a model to localise itself, plan its trajectory and find obstacle free paths. Creating maps of static environments has been widely studied for a long time [6]. However, dynamic scenarios, where the world is constantly changing and uncertainty grows with time, are still an open problem.

Some authors try to handle these dynamics by finding the most static landmarks and filtering out the ones that change over time [7]. Another approach involves tracking these "moving" landmarks and labelling them as dynamic [8]. In general, these approaches can handle some problems of navigating in a dynamic environment, but they cannot deal with long-term changes to the structure of the environment.

Other approaches never assume the map to be complete and perform continuous mapping, adding new features to the map with every observation [9], [10], [11]. In these approaches, the key problem is managing map size, especially in long term scenarios where the robot might be making new observations for several weeks or months.

To tackle the long-term challenges of robust navigation in dynamic scenarios, some approaches gather and maintain different temporal representations simultaneously and choose the best one according to its consistency with the current perception of the world (e.g. [9], [12]). However, these approaches have high memory and computational costs.

To reduce computational cost, many authors have tried taking these approaches to a discrete, topological level. where most representations use visual appearance for place recognition [13], [14]. However, these approaches present a decrease in robustness when facing long-term changes [15], as again they are prone to error when features appear and disappear over time. In [3], [4] dynamic models of the topological space that explicitly represent the environment changes and try to identify patterns by means of the Fourier transform are presented, for both localisation (node level) and navigation (transitions between nodes). This ability of pattern identification allows for state prediction, which as shown in [4] can improve navigation performance. However, these approaches are limited to higher level planning at the topological level and there is no model that can be used for low-level navigation on the node transitions.

Other authors have looked into metric level representations with state prediction abilities. In [16] the authors propose a new representation that models occupancy grid maps in the wavelet space in order to optimize the amount of information that has to be processed for path planning, and [17] presents a representation of the environment which models transitions of dynamic objects in the environment, by learning motion patterns from the temporal signal of occupancy in a cell.

The approach presented in this paper presents an occupancy grid map where each cell in the map is enhanced with a spectral model [2] that enables the prediction of the cell's state at specific times. We show how localisation and navigation are improved using such a representation compared to a static occupancy grid map, which is still the most common approach.

\section{SYSTEM DESCRIPTION}

A major drawback of the previously proposed dynamic mapping techniques is that they are tailored to particular representations that only work for specific system architectures. The objective of this work was to develop an environment representation that is general enough to allow its use with most of the environment models used in robotics. Moreover, we wanted the system to be easy to use by other researchers, and therefore, it was implemented as a module that is compatible with the navigation stack [18] of the Robot Operating System (ROS), which is considered a standard in robotics nowadays. The software presented here is freely available as a component of the STRANDS system [19].

In principle, the framework allows to keep several spatiotemporal models that can be used for localisation simultanneously as proposed by [9], [12]. Building a separate map of the current environment layout allows to postpone the decision on which of the global spatio-temporal models will be updated similarly to the experience-based approach in [12]. This would not be possible with a classic continuous SLAM approach. In this work, we use only one global spatiotemporal representation and if the currently built map is detected as anomalous it is simply rejected.

\section{A. Continuous Mapping with the ROS navigation stack}

The traditional configuration of the navigation stack has four main components, see Figure 3 (left): the robot, which provides all the necessary sensory input and coordinate system transformations; the map server, which provides the map used by the localisation and planning systems, where this map is usually created in a previous stage; the $A M C L$ localisation system, which provides position estimates in the map coordinate frame using the sensory input from the robot; and a move base motion planner that uses the map, position estimate and sensor information to plan the robot's motion.

One of the key requirements of long-term operation is to keep the environment models up-to-date. In our architecture, a traditional SLAM-based method is used to create a completely new map every time the robot performs a patrol run and integrate this single map into the proposed spatiotemporal representation. The ability to update the environment map is achieved through a minor modification of the ROS navigation stack, see Figure 3 (right). The main changes 

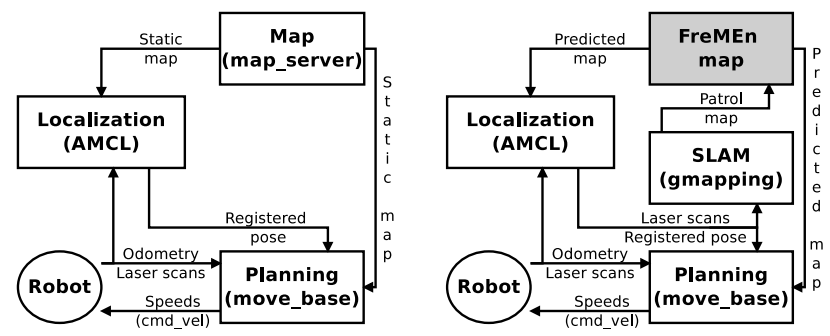

Fig. 3: Classic and proposed ROS navigation stack.

are the continuous mapping component and the spatiotemporal representation, called the FreMEn map, which provides time-specific maps to the other system modules. Continuous mapping is achieved by setting up the gmapping module to work in parallel with the rest of the navigation stack during robot operation and creating a new 'patrol' map for each session, see Figure 3. However, the SLAM-based gmapping implementation is subject to a slight localisation drift and running it as a completely separate process would cause its map to diverge from the global 'FreMEn' one, which would make its integration into the FreMEn map impossible. To prevent this drift, we inject the output of the AMCL position estimation in the odometry input of the gmapping module. This position injection ensures that the individual cells of the 'patrol' and FreMEn maps are wellaligned with each other and that the cell differences are caused by environment changes and not by localisation drift. This makes integration of the 'patrol' map into the global one a straightforward process.

\section{B. FreMEn 2D Grid}

The idea of the FreMEn map is based on the observation that many of the environment states are not changing chaotically and the nature of their dynamics can be learned from repeated observations. Thus, the FreMEn map is a $2 \mathrm{~d}$ occupancy grid that does not represent the uncertainty of the individual cells by a constant probability, but as a function of time, which is estimated from re-observations of the cell occupancy over long time periods. The FreMEn map can integrate local $2 \mathrm{~d}$ grids created at different times ('patrol maps') into a global spatio-temporal representation that captures not only the spatial layout of the environment, but also the persistence and cyclic behaviour of its changing states.

From an architectural point of view the FreMEn map component provides the $2 \mathrm{~d}$ environment maps in the same way as the original ROS map server. However, unlike the map server, which can load, save and transmit static maps only, the FreMEn map generates time-dependent maps that reflect the expected environment state at the time of robot operation.

Although the model update step can be performed at any time, the mapping process should be long enough to filter out fluctuations in the environment, and the map update should only happen when the robot is not moving to avoid anomalous maps.

\section{Anomaly detection}

Continuous mapping is exposed to two significant threats. First, the gmapping method can fail and produce an incorrect map, which, when integrated into the FreMEn map, might corrupt the entire spatio-temporal representation. The second threat is more subtle: due to the environment changes, sensor noise and localisation inaccuracies, exact registration of the recently-gathered 'patrol' maps with the FreMEn grid is not absolutely precise. This introduces a certain amount of noise every time a new map is integrated into the global one. As the noise accumulates, the global map might become less and less accurate over time, which might lead to its destruction.

Both effects exhibit themselves at the moment when a new map is integrated into the FreMEn grid. Therefore, the FreMEn map checks how much a new map conforms to the map predicted for that particular time by calculating the number of cells with incorrectly predicted occupancy. If the ratio of these cells in the new map is too high we reject this map as an outlier.

\section{FRequency Map Enhanced ocCUPANCY GRID}

The spatio-temporal representation at the core of our approach is a $2 \mathrm{~d}$ occupancy grid that models the occupancy of each cell by a probabilistic function of time. This probabilistic function consists of two distinct components: persistence and periodicity. The persistence component acts as a short-term memory that represents the expectation that the cell state did not change since the last observation if the observation was performed recently. The periodicity component is related to the fact that from a long-term perspective (days to months), some of the environment states might be influenced by hidden periodic processes.

The idea of identifying periodic patterns of binary environment states via the Fourier Transform and using them for future predictions was originally presented in [2]. In our version of FreMEn, we represent the periodic behaviour of each cell by its sparse frequency spectrum, which is a set $\mathcal{A}$ of complex numbers $\alpha_{k}$. These correspond to the set $\Omega$ of modeled periodicities $\omega_{k}$ that might be present in the environment. The persistence of each state is represented by the mean time between state transitions $\tau$ and the time and value of the last observation $t_{l}$ and $s\left(t_{l}\right)$. Moreover, we store each cell's number of observations $n$ and the cell's mean occupancy $\mu$. When a 'patrol' map is integrated in the FreMEn map, each cell representation is updated as follows:

$$
\begin{aligned}
\mu & \leftarrow \frac{1}{n+1}(n \mu+s(t)), \\
\alpha_{k} & \leftarrow \frac{1}{n+1}\left(n \alpha_{k}+s(t) e^{-j t \omega_{k}}\right) \quad \forall \omega_{k} \in \Omega, \\
\frac{1}{\tau} & \leftarrow \frac{1}{n+1}\left(\frac{n}{\tau}+\frac{\left|s(t)-s\left(t_{l}\right)\right|}{t-t_{l}}\right), \\
s\left(t_{l}\right) & \leftarrow s(t) \\
t_{l} & \leftarrow t, \\
n & \leftarrow n+1,
\end{aligned}
$$

where $s(t)$ is the occupancy of a given cell in the 'patrol' map created at time $t$. The proposed update step is analogous to incremental averaging, where the absolute values of $\left|\alpha_{k}\right|$ 
correspond to the average influence of a periodic process (with a frequency of $\omega_{k}$ ) on the values of $s(t)$. Note that the size of the representation of the state (i.e. the number of elements in $\mathcal{A}$ ) is independent of the number of observations, which means that the memory requirements of the proposed representation do not grow with time.

To predict the value of state $s(t)$ for a future time $t$, we first sort the set $\mathcal{A}$ descendingly according to the absolute values $\left|\alpha_{k}\right|$. Then, we extract the first $m$ elements $\alpha_{l}$ along with their corresponding frequencies $\omega_{l}$ and calculate the state's probability over time as

$$
p(t)=s\left(t_{l}\right) e^{\frac{t_{l}-t}{\tau}}+f(t)\left(1-e^{\frac{t_{l}-t}{\tau}}\right),
$$

where

$$
f(t)=\varsigma\left(\mu+\sum_{l=1}^{m}\left|2 \alpha_{l}\right| \cos \left(\omega_{k} t+\arg \left(\alpha_{l}\right)\right)\right),
$$

where $\varsigma($.$) ensures that p(t) \in[0,1]$. Note that for preditions which immediatelly follow the last observation, i.e. $\mid t-$ $t_{l} \mid<<\tau$, the expression $e^{\frac{t_{l}-t}{\tau}}$ is close to 1 , which means that the expected occupancy would be the same as the one recently observed. If we use Equation 2 to predict further into the future, i.e. $\left|t-t_{l}\right|>>\tau$, the expression $e^{\frac{t_{l}-t}{\tau}}$ is close to 0 , which supresses the effect of the last observation on $p(t)$ and emphasizes $f(t)$, which represents the behaviour of the modelled cell from a long-term perspective.

Note that the choice of $m$, which determines how many periodic processes are considered, and $\Omega$, which determines the periods of the potential cyclic processes, are crucial for prediction performance. In our case, $m$ was set to 2 and the $\omega_{k}=\frac{2 \pi k}{86400}$, where $k \in\{1,2, \ldots 24\}$, which allows to model periodicities ranging from 1 hour up to 1 day.

The model does not expect that the behaviour of the environment is strictly periodic, but it reflects the fact that probabilities of the environment states are influenced by cyclic events. For example, the FreMEn can reflect the fact that human presence in a research office causes doors of certain cupboards to be more likely open during the weekdays than during nights, see Figure 2.

\section{EXPERIMENTS}

To evaluate the utility of the proposed dynamic map, we used data gathered during several days of routine autonomous operation of a mobile robot at the Lincoln Centre for Autonomous Systems. The SCITOS-G5 mobile robot (see Figure 4) regularly patrolled a large open-plan office every ten minutes while recording data from its odometry, RGB-D and laser range-finder sensors. Its navigation was based on the ROS navigation stack, which used our FreMEn 2D grid instead of the traditional map server. To achieve autonomous operation, the robot uses a precise visual servoing method for reliable docking to its charging station [20]. Our evaluation was based on three criteria: localization accuracy, navigation efficiency and map quality. To evaluate localization accuracy, we covered part of the environment with an external localization system, which provided us with a ground truth of

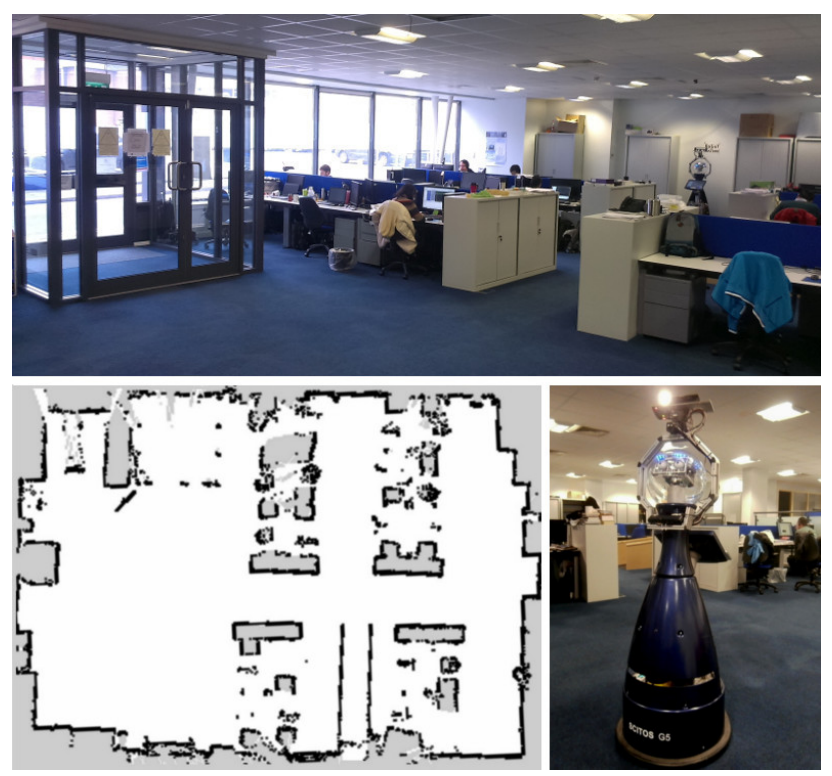

Fig. 4: Overview and 2D occupancy map of the Witham Wharf office.

the robot position with millimetre precision. To quantify the efficiency of the robot navigation, we measured the time it took to perform a patrol where the robot had to visit five different locations. We also measured the times it took for the robot to navigate through a narrow area that exhibited regular changes. To assess the quality of the built maps, we quantified the amount of noise in the maps.

\section{A. Localisation accuracy}

To evaluate the accuracy of self-localization, we installed an independent localisation infrastructure at the Witham Wharf office, comprising two ceiling-mounted fish eye Kodak PixPro SP360 cameras, a large circular marker on top of the robot and another set of markers close to the robot's charging station. While the marker on the robot's top was used to determine its $x$ and $y$ position, the markers at the charging station area allowed for precise, independent estimation of the robot heading. Detection and position estimation of the markers, localisation system calibration and coordinate system setup was based on a freely-available, open-source method presented in [20]. To ensure millimetre accuracy of the localisation system, we had to use rather large markers as suggested by the mathematical model of the system [20], see Figure 5. We selected approximately 2000 images in 20 different image sequences captured by the overhead cameras and established the positions of the robot. To avoid potential accuracy drop-off caused by the use of the wide-angle lens cameras, the selected images have the robot position close to the center of the image.

The individual sequences captured the movement of the robot through a $1.5 \mathrm{~m}$ wide corridor outlined by eight storage cupboards. These cupboards are used by the research staff of the office and some of the cupboard doors are typically open during the day and closed at night. The cupboards are $0.5 \mathrm{~m}$ 


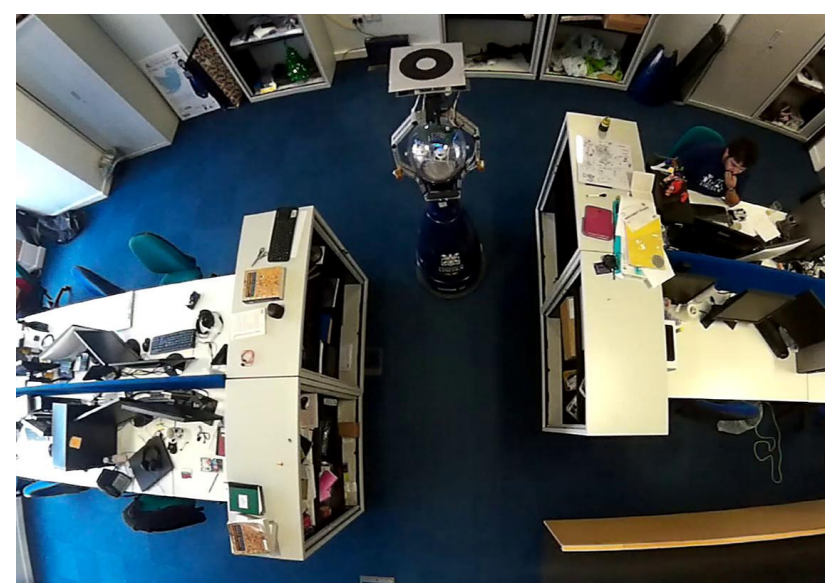

Fig. 5: Example image captured by the ceiling-mounted camera of the external localisation system. The circular marker on top of the robot is used to provide the ground truth for robot self-localization.

deep, so when a cupboard door is left open, the corridor appears to be $2 \mathrm{~m}$ instead of $1.5 \mathrm{~m}$ wide and its center appears to be offset by $0.25 \mathrm{~m}$. Thus, when moving through this corridor, the discrepancy of the 2D map with the perceived environment state might negatively affect the accuracy of robot self-localization. In our case, the $20 \mathrm{~m}$ range of the robot laser rangefinder ensures that it will almost always perceive areas that did not change, which should keep the position estimate accurate. However, if the range of the laser sensor was shorter, e.g. when using a Hokyo URG04, then the localization accuracy would be affected severely.

To estimate the impact of the environment change and sensor range on the localization precision, we processed laser, odometry and ground truth data from 20 different passes of the robot through the monitored corridor. To emulate the limited range of the laser rangefinder, we trimmed the laser data at different lengths. Using the trimmed data from 20 different runs, we performed standard ROS-based AMCL localisation on the 'static', 'averaged' and 'predicted' $2 \mathrm{~d}$ maps and compared the robot positions to the ground truth from the overhead cameras. The results shown in Figure 6 indicate that use of the time-specific, predicted maps significantly improves the localization precision if the range of the laser rangefinder is lower than the overall map size. If the rangefinder provides a complete overview of the operational environment, the reduction of the position estimation error is only marginal. However, a small difference in localization precision can have a significant impact on the efficiency of the robot navigation and quality of the constructed maps.

\section{B. Navigation efficiency}

To evaluate the navigation efficiency, we processed navigation statistics of 60 different patrol runs. During each patrol, the robot undocked from its charging station, visited several different locations in the office (see Figure 2) and returned to recharge. The data from each patrol run contains the robot's average speed and the number of 'recovery events'
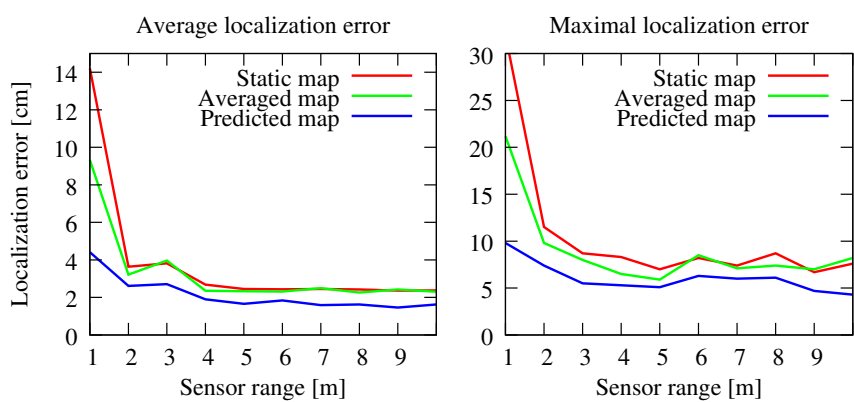

Fig. 6: Localization error for different ranges of the laser scanner and different types of the maps. Predicting a map for a particular time improves localization accuracy, although the improvement is only marginal for long-range sensors.

where standard navigation behaviour failed and the robot had to perform custom recovery behaviours in order to avoid a collision and proceed with its patrol. The gathered navigation statistics were divided into three groups of 20 patrols each. The first group contained patrols at weekends, where the number of environment changes in the office is rather low. The second group contained patrols from weekday afternoons, where the robot used an 'averaged' map, which slowly adapts to the observed changes. The third group contained patrols from weekday afternoons, where the robot used a 'predicted', time specific map. In the latter two cases, the number of environment changes introduced by $\sim 20$ researchers of the open-plan office was relatively high. Table I indicates that in a static environment, the robot

TABLE I: Navigation statistics

\begin{tabular}{lrrrr}
\hline Environment & Static & \multicolumn{2}{c}{ Changing } \\
Map & Static & Static & Predicted \\
\hline Average speed & {$\left[\frac{m}{s}\right]$} & 0.21 & 0.16 & 0.18 \\
Recovery events & {$[-]$} & 3 & 21 & 13 \\
\hline
\end{tabular}

could navigate efficiently even when using a static map, but as soon as the environment began to change, the navigation efficiency was affected negatively. However, this negative effect was lowered slightly through the use of the proposed map, which explictly represents the environment changes.

\section{Map quality}

This experiment evaluates the effect of the anomalous map detection mechanism. This mechanism verifies whether a newly created map conforms with the representation that was gathered so far, which allows to reject corrupt or otherwise incorrect maps. To verify the utility of the anomaly detection mechanism, we replayed laser and odometry data from 70 consecutive patrols with the anomaly detection component being deactivated and compared the resulting spatio-temporal representation with the one built while the anomaly detection was used to reject potentially corrupted maps. Figure 7 shows the percentage of incorrectly predicted cells in the consecutively created maps. The figure shows that at a certain point 
(run 36), integration of an incorrect map corrupts the FreMEn grid, which breaks the map update process. However, the
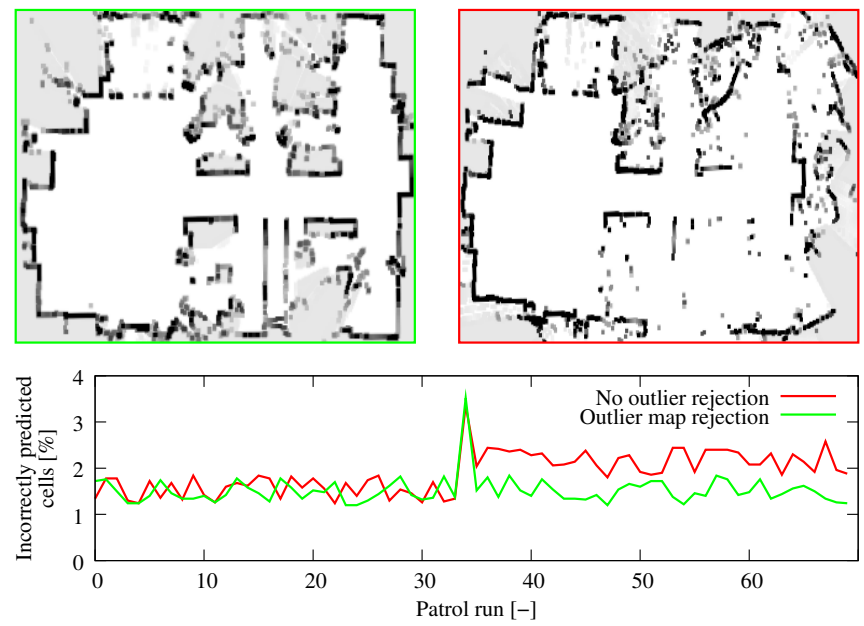

Fig. 7: Effect of anomalous map detection and rejection. The peak in the graph indicates mapping failure and consequent discrepancy of the individual maps. The two maps show the resulting models with and without anomaly rejection.

anomalous map rejection mechanism prevents this situation and the map update process continues to produce a faithful 2D environment model.

\section{CONCLUSION}

We presented an approach for persistent localization and mapping in changing environments. First, we show that the ability to update the environment model does not require introduction of custom modules to the ROS navigation stack. Instead, the navigation stack can be augmented by an alternative gmapping module that builds a new map every time the robot navigates around its operational environment. To ensure that the new map is consistent with the previously built model, we propose to use the $A M C L$ module position estimation as virtual odometry for gmapping. Second, we demonstrate that maps of the individual navigation runs can be integrated into a spatio-temporal model that captures the persistence and periodicity of the environment changes. This spatio-temporal environment representation, which explicitly models the environment dynamics, is used to predict timespecific maps, which serve our robot both for localization, path-planning and navigation.

Our experimental evaluation, based on data gathered over the course of several weeks, shows that using the model's predictive capabilities improves the accuracy of robot localization and efficiency of navigation. The tests indicate that the proposed environment model is especially beneficial for mobile robots that do not have a complete overview of their environment, e.g. due to the limited sensor range such as when operating outdoors or in large warehouses.

While encouraging, the experiments were too short to demonstrate that the proposed method enables life-long autonomous operation in changing environments. Therefore, as part of our project goals [19], we plan the deployment of the method on a mobile robot that will operate at a large care home for a period of four months.

Moreover, we plan to extend the anomaly detection mechanism so that an anomalous map would not be rejected, but stored as an alternative map. This alternative map would represent a hypothesis that that the map change was caused by an actual environment change rather than mapping malfunction. This could result in additional robustness of the system to significant environment changes.

\section{REFERENCES}

[1] M. Quigley, K. Conley, B. P. Gerkey, J. Faust, T. Foote, J. Leibs, R. Wheeler, and A. Y. Ng, "ROS: an open-source robot operating system," in ICRA Workshop on Open Source Software, 2009.

[2] T. Krajník, J. P. Fentanes, G. Cielniak, C. Dondrup, and T. Duckett, "Spectral analysis for long-term robotic mapping," in Proc. ICRA, 2014.

[3] T. Krajník, J. P. Fentanes, O. M. Mozos, T. Duckett, J. Ekekrantz, and M. Hanheide, "Long-term topological localization for service robots in dynamic environments using spectral maps," in Proc. IROS, 2014.

[4] J. Pulido Fentanes, B. Lacerda, T. Krajník, N. Hawes, and M. Hanheide, "Now or later? predicting and maximising success of navigation actions from long-term experience," in Proc. ICRA, 2015.

[5] T. Krajník, M. Kulich, L. Mudrová, R. Ambrus, and T. Duckett, "Where's Waldo at time t? using spatio-temporal models for mobile robot search," in Proc. ICRA, 2015.

[6] S. Thrun, "Robotic mapping: A survey," Exploring artificial intelligence in the new millennium, pp. 1-35, 2002.

[7] D. Wolf and G. Sukhatme, "Mobile robot simultaneous localization and mapping in dynamic environments," Autonomous Robots, 2005.

[8] L. Montesano, J. Minguez, and L. Montano, "Modeling dynamic scenarios for local sensor-based motion planning," Autonomous Robots, vol. 25, no. 3, pp. 231-251, 2008.

[9] P. Biber and T. Duckett, "Experimental analysis of sample-based maps for long-term SLAM," Int. J. Robotics Research, 2009.

[10] M. Milford and G. Wyeth, "Persistent navigation and mapping using a biologically inspired SLAM system," Int. J. Robotics Research, vol. 29, no. 9, pp. 1131-1153, 2010.

[11] K. Konolige and J. Bowman, "Towards lifelong visual maps," in Proc. IROS, 2009

[12] W. Churchill and P. Newman, "Practice makes perfect? Managing and leveraging visual experiences for lifelong navigation," in Proc. ICRA, 2012.

[13] T. Krajník, S. Pedre, and L. Přeučil, "Monocular navigation system for long-term autonomy," in Proc. ICAR, 2013.

[14] C. Cadena, D. Gálvez-López, J. D. Tardós, and J. Neira, "Robust place recognition with stereo sequences," IEEE Trans. on Robotics, 2012.

[15] F. Dayoub, G. Cielniak, and T. Duckett, "Long-term experiments with an adaptive spherical view representation for navigation in changing environments," Robotics and Autonomous Systems, 2011.

[16] M. Yguel, O. Aycard, and C. Laugier, "Wavelet occupancy grids: a method for compact map building," in Field and Service Robotics. Springer, 2006, pp. 219-230.

[17] T. Kucner, J. Saarinen, M. Magnusson, and A. Lilienthal, "Conditional transition maps: Learning motion patterns in dynamic environments," in Proc. IROS, 2013.

[18] E. Marder-Eppstein, E. Berger, T. Foote, B. Gerkey, and K. Konolige, "The office marathon: Robust navigation in an indoor office environment," in Proc. ICRA, May 2010, pp. 300-307.

[19] "Spatio-temporal representations and activities for cognitive control in long-term scenarios," [Cit: 2016-02-29]. [Online]. Available: http://www.strands-project.eu

[20] T. Krajník, M. Nitsche, J. Faigl, P. Vaněk, M. Saska, L. Přeučil, T. Duckett, and M. Mejail, "A practical multirobot localization system," J. Intelligent \& Robotic Systems, vol. 76, no. 3-4, pp. 539-562, 2014. 